\title{
Comentario editorial sobre primera nefrectomía transvaginal híbrida por cáncer renal
}

En referencia al trabajo de este número que aparece en las páginas 280-283

René Sotelo Noguera

Centro de Cirugía Robótica y de Invasión Mínima. Instituto Médico La Floresta. Caracas, Venezuela

$\mathrm{E}$ 1 desarrollo de NOTES (Natural Oriffice Transluminal Endoscopic Surgery) en urología no ha sido fácil debido al tamaño de las piezas operatorias, la necesidad de reconstrucción y al enfrentamiento en general con grandes pedículos vasculares que se hacen de difícil manejo con los instrumentos disponibles en la actualidad y mas aún cuando la intervención supone ser realizada tan solo a través de los orificios naturales. Es por esto que en la búsqueda de la cosmesis y menor morbilidad, surgió la opción de la cirugía a través de un orificio escondido en la cicatriz umbilical como abordaje único, que después de un consenso recibió la denominación de LESS (Laparo-Endoscopic Single Site); con el tiempo ha venido resurgiendo la opción de NOTES en una forma hibrida, que consiste en utilizar un orificio natural y además apoyarse con trocares a través de la pared abdominal, siendo esta la forma mas cercana a NOTES de uso clínico que se ha desarrollado en urología.

Desde 1993 la vagina había sido descrita en nefrectomías, fundamentalmente para la extracción de la pieza operatoria por Breda $^{1}$; e incluso antes de la denominación del abordaje NOTES, Gettman en el 2002 exploró la posibilidad de hacer nefrectomías porcinas solamente a través de la vagina siendo exitoso el procedimiento en uno de seis $\operatorname{casos}^{2}$ y en el mismo año Gill realiza un abordaje y disección laparoscópicos con tres trocares finalizando la extracción por vía transvaginal en humanos ${ }^{3}$.

En este artículo de Alcaraz y también en el artículo de hace algunos meses de Branco en Brazil, se discute la posibilidad de usar la vagina para la colocación en el caso de Alcaraz de un endoscopio rígido con punta flexible y en el caso de Branco un endoscopio flexible tipo gastroscopio ${ }^{4}$. Es interesante, porque estas perspectivas nos inducen a buscar las opciones tanto de introducción de ópticas como de pinzas a través de la vagina que puedan ayudar en la disección, no solamente utilizando la vagina para la extracción de la pieza operatoria sino también como vía de abordaje quirúrgico.

Con la evolución de las diferentes técnicas laparoscópicas, se observa de manera llamativa la migración en la ubicación de los trocares de la pared abdominal para asistir esta cirugía. En el trabajo de Branco se colocaron dos trocares de $5 \mathrm{~mm}$, uno en la cicatriz umbilical y otro generalmente cerca al reborde costal, él introduce el endoscopio por la vagina y en algunos momentos de la cirugía a través de uno de los trocares de $5 \mathrm{~mm}$ abdominal para ayudarse en la disección; a diferencia del Alcaraz quien usa el endoscopio EndoEye 10mm con punta deflectable y dos trocares en la pared abdominal fundamentalmente para tratar de mantener el principio de la triangulación, sin utilizar la cicatriz umbilical.

Este artículo de Alcaraz será trascendental en la evolución de estos procedimientos, fundamentalmente por el uso de la vía vaginal. En este momento algunos podrán argumentar que la diferencia entre esta nefrectomía que propone Alcaraz y la nefrectomía laparoscópica convencional en la cual se colocan los tres trocares abdominales y se extrae la pieza operatoria por la vagina, es simplemente un trocar menos en la pared abdominal.

La real diferencia no radica en un trocar menos, la constituye el inicio del uso de la vagina adicional a la extracción, fundamentalmente como un puerto de trabajo.

Con el advenimiento de investigaciones en el tema, probablemente la ubicación de los trocares van a ser migrados hacia la cicatriz umbilical. Posiblemente las nefrectomias serian realizadas con un trocar unico multivalvular, o varios trocares de 5 $\mathrm{mm}$ dentro de la cicatriz umbilical y un trocar multivalvular en la vagina, con lo cual la nefrectomía transvaginal sería asistida exclusivamente por un trocar ubicado dentro de la cicatriz umbilical mejorando aún los resultados cosméticos. 
También hay intentos interesantes del grupo de Cadeddu quienes introducen retractores mantenidos a traves de imanes en la pared abdominal, que pueden ayudar a separar ${ }^{5}$. Estos ingenios y algunos otros, evidencian la evolución constante en la busqueda de optimizar la tecnologia a las nuevas tendencias y no tratar de adaptar las viejas herramientas a los nuevos requerimientos. Estamos sólo en el principio, el futuro sera de mejores intrumentos articulados, sistemas de retraccion internos libres de soporte externos, endoscopios con canales articulables independientes, y finalmente plataformas robóticas para el manejo de estos minúsculos tentáculos que se abrirán dentro de las cavidades restableciendo internamente el viejo principio de la triangulación.

Finalmente son imprescindibles estudios comparativos entre laparoscopia convencional, LESS, NOTES y NOTES híbrido, evaluando los parametros tradicionales pero además debe hacerse énfasis en nuevas escalas mas precisas para la medición de convalecencia y cosmesis.

\section{REFERENCIAS}

1. Breda G, Silvestre P, Guinuta A, Xausa D, Tamai A, Gherardi L. Laparoscopic nephrectomy with vaginal delivery of the intact kidney. Eur Urol. 1993;24(1):116-117.

2. Gettman MT, Lotan Y, Napper CA, Cadeddu JA. Transvaginal laparoscopic nephrectomy: development and feasibility in the porcine model. Urology 2002;59(3):446-450.

3. Gill IS, Cherullo EE, Meraney AM, Borsuk F, Murphy DP, Falcone T. Vaginal extraction of the intact specimen following laparoscopic radical nephrectomy. J Urol 2002 Jan;167(1):238241.

4. Branco AW, Branco Filho AJ, Kondo W, Noda RW, Kawahara N, Camargo AA et al. Hybrid Transvaginal Nephrectomy. 1: Eur Urol. 2008 Jun;53(6):1290-1294.

5. Raman JD, Bergs RA, Fernandez R, Bagrodia A, Scott DJ, Tang SJ, Pearle MS, Cadeddu JA. Complete Transvaginal NOTES Nephrectomy Using Magnetically Anchored Instrumentation. J Endourol. 2009 Feb 4.

Correspondencia autor: Dr. René Sotelo Centro de Cirugía Robótica y de Invasión Mínima Instituto Médico La Floresta. Caracas, Venezuela Tel.: 582122851015

E-mail autor: urologialaparoscopica@gmail.com Información artículo: Editorial Trabajo recibido: marzo 2008 